\title{
Perfil de usuários e percepção sobre a qualidade do atendimento nutricional em um Programa de Saúde para a Terceira Idade
}

\author{
Users' profileand perceptionabat quality of nutritiond careina HealthProgramfor the Edelly
}

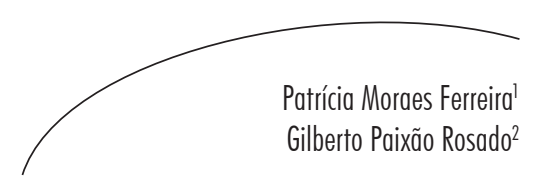

Resumo

Objetivou-se analisar o perfil dos usuários de um serviço de nutrição e a percepção dos mesmos sobre a qualidade do atendimento, em uma pesquisa quali-quantitativa, utilizando entrevistas semiestruturadas, conduzidas logo após o atendimento nutricional a 32 idosos de um programa para a terceira idade no município de Viçosa - MG. Predominou o sexo feminino, com idades entre 60 e 78 anos; a hipertensão arterial foi a doença de maior ocorrência; a maioria está satisfeita com o atendimento; $44,8 \%$ têm dificuldades para mudar a alimentação e 100\% foram otimistas quanto ao resultado do acompanhamento nutricional; a maioria é portadora de doenças crônicas não-transmissíveis, possui autonomia, compreende o serviço como instrumento de auxílio na qualidade de vida e associa a satisfação com o atendimento a aspectos de cuidado e atenção. Estimular pesquisas com programas de nutrição na terceira idade se torna cada vez mais necessário, para que se busque qualidade nutricional e melhores estratégias de atendimento aos idosos.

\section{Abstract}

This study analyzes the profile of users of nutrition services and their perception about the quality of care, in qualitative and quantitative research, using semi-structured interviews, conducted shortly after the nutritional care to 32 elderly of a program for the aged in Viçosa city, state of Minas Gerais, Brazil. The majority were female, aged between 60 and 78 years old; hypertension was the most frequent disease; most were satisfied with the service; $44.8 \%$ reported some difficulties in changing their diet and $100 \%$ were optimistic about the outcome of counseling; most have chronic non-communicable diseases, are autonomous, understand the service as an instrument of quality of life and derives satisfaction from the care and attention. Encouraging researches on nutrition programs for the elderly becomes increasingly necessary, in order to seek nutritional quality and better strategies of care for the elderly.

1 Programa de Pós-Graduação em Saúde Coletiva, Faculdade de Medicina de Botucatu. Universidade Estadual Paulista "Júlio de Mesquita Filho". Botucatu, SP, Brasil.

2 Departamento de Nutrição e Saúde. Universidade Federal de Viçosa. Viçosa, MG, Brasil.

Palavras-chave: Percepção. Serviços de Saúde para Idosos. Programas de Nutrição. Assistência à Saúde.

Key words: Perception. Health Services for the Aged. Nutrition Programs. Delivery of Health Care. 


\section{INTRODUÇÃO}

Envelhecer é um processo natural, caracterizado por uma redução progressiva da capacidade auditiva, funcional, e alterações das funções metabólicas, que não necessariamente acarretarão ocorrência de enfermidades. ${ }^{1} \mathrm{O}$ frequente aumento das doenças crônicas, a utilização de medicamentos, as mudanças fisiológicas inerentes à idade que interferem no apetite, na ingestão e absorção de nutrientes, e os problemas sociais e econômicos são os principais fatores que dificultam a manutenção de um estado nutricional adequado no indivíduo idoso. ${ }^{2,3}$

Uma das formas de promover melhoria na qualidade de vida dos idosos é desenvolver programas de saúde que, segundo a Organização Mundial da Saúde (OMS), têm como base a comunidade, implicam promoção da saúde do idoso e devem ter a nutrição como uma das áreas prioritárias. ${ }^{4}$ A boa nutrição é um dos fatores que levam a um envelhecimento sadio, sendo que a manutenção de estado nutricional adequado não deve ser entendida como estratégia para obter maior sobrevida, mas como um fator que interfere positivamente para que um número maior de pessoas se aproxime do seu ciclo máximo de vida. ${ }^{5}$

$\mathrm{O}$ poder que o indivíduo tem de avaliar, decidir, escolher, sobretudo o que lhe diz respeito, chama-se autonomia. ${ }^{6} \mathrm{O}$ documento "Envelhecimento ativo: uma política de saúde", da OMS,? adotou o termo "envelhecimento ativo" para defender a visão de que esse processo, para ser vivenciado de maneira positiva, deve ser acompanhado de oportunidades contínuas de saúde, participação e segurança. Também destaca que “a palavra 'ativo' refere-se à participação contínua nas questões sociais, econômicas, culturais, espirituais e civis, e não somente à capacidade de estar fisicamente ativo ou de fazer parte da força de trabalho".

Alguns estudos fizeram menção à ocorrência cada vez maior de inclusão social dos idosos, que constroem aos poucos seus espaços de socialização, principalmente por meio dos grupos de terceira idade. ${ }^{8,9}$ A maioria dos idosos que frequentam grupos de terceira idade têm uma visão positiva em relação ao envelhecimento, interpretando esse momento como sendo oportuno para a construção de algo novo. ${ }^{8}$

O Programa Municipal da Terceira Idade (PMTI), do município de Viçosa, é exemplo de um serviço. Conta com 2.100 idosos cadastrados e foi instituído em 1997, em parceria com o Núcleo de Saúde Pública (NUSP), do Departamento de Nutrição e Saúde (DNS) da Universidade Federal de Viçosa e Prefeitura Municipal de Viçosa. Nesse programa são realizadas atividades educativas como palestras, oficinas e aulas de alfabetização e atividades de prevenção de doenças como atendimentos multiprofissionais, objetivando melhorar a qualidade de vida dos participantes. As principais atividades desenvolvidas são: recreação, oficinas, atividade física, atendimento médico, de enfermagem, odontológico, fisioterapêutico e nutricional.

Diante do contexto atual de transição demográfica, epidemiológica e nutricional, e consequente preocupação de entidades com a promoção da saúde, criam-se cada vez mais programas/serviços de saúde que objetivam melhorar a qualidade de vida na terceira idade. Como resultado, surge uma clientela específica: indivíduos que irão usufruir desses serviços, tornando-se membros ativos nesse processo, que devem, assim, assumir o papel de se manifestar em relação a esses serviços, analisando a qualidade dos mesmos, que é determinada pelas experiências individuais junto ao programa.

O termo "qualidade", no que se refere à prestação de cuidado, relaciona-se a um vasto espectro de características como: efetividade nos resultados, eficiência, equidade, aceitabilidade, acessibilidade e adequabilidade. ${ }^{10}$ Portanto, se faz necessário definir qual/quais aspectos da qualidade serão levados em conta pelo usuário para a posterior avaliação. ${ }^{11}$

Alguns estudos incorporaram subjetividade ao processo de análise/avaliação de serviços, ${ }^{12-14}$ mas 
a ênfase em serviços como o de acompanhamento nutricional é pouco explorada nas pesquisas brasileiras, o que requer aprofundamento no estudo de programas como o PMTI, que contribuem para a melhoria da qualidade de vida na terceira idade.

Sendo assim, este trabalho teve como objetivo analisar o perfil socioeconômico e de saúde dos idosos atendidos pelo serviço de Nutrição do PMTI, bem como analisar a percepção dos mesmos sobre a qualidade do atendimento nutricional, enfatizando os aspectos de qualidade relacionados ao atendimento e seus resultados, satisfação do usuário, cuidado, comunicação interpessoal, tempo de dedicação dos estagiários e nutricionista à consulta e resultados na expectativa com a saúde pelo paciente após o acompanhamento nutricional.

\section{METODOLOGIA}

Trata-se de estudo transversal, de abordagem quali-quantitativa, cuja coleta de dados ocorreu no período de fevereiro a junho de 2009. Utilizaram-se como técnica de coleta de dados entrevistas semiestruturadas, com perguntas abertas e fechadas, conduzidas logo após o atendimento nutricional aos idosos participantes do Programa Municipal da Terceira Idade (PMTI), no município de Viçosa-MG.

De acordo com o banco de dados do programa nos anos de 2007 e 2008, o atendimento nutricional médio anual foi de 78 idosos. Foram selecionados os idosos que aceitaram participar do estudo após esclarecimentos a respeito dos objetivos da pesquisa e que se mostraram em condições de responder aos questionários. A coleta de dados foi realizada após assinatura do termo de consentimento livre e esclarecido, e a unidade amostral foi composta de 32 idosos de 60 anos ou mais, cadastrados no PMTI, do município de Viçosa-MG, e que foram atendidos pela nutricionista do programa e estagiários do último período do Curso de Nutrição da Universidade Federal de Viçosa (UFV).
As análises fundamentaram-se em dois planos: a) o plano de dimensão subjetiva, quando determinadas questões se referiam a experiência e individualidade dos sujeitos, sendo utilizados como material básico os discursos dos idosos e a perspectiva teórica adotada para interpretá-los; b) o plano de dimensão objetiva, para verificação dos dados quantitativos, sendo feitas análises de verificação de frequência simples e valor absoluto, utilizando-se o software SPSS (Statistical Package for the Social Sciences), versão 15.0.

Este trabalho foi aprovado pelo Comitê de Ética em Pesquisa com Seres Humanos (Of. Ref. $n^{\circ}$ 005/2009) da Universidade Federal de Viçosa-MG.

\section{RESULTADOS}

0 perfil dos idosos que buscam 0 atendimento nutricional do Programa Municipal da Terceira Idade

A amostra constituiu-se de 32 idosos, com predomínio do sexo feminino (84,4\%) sobre o masculino $(15,6 \%)$. A idade mínima foi de 60 anos, média de 68,9, mediana de 69 e máxima de 78 anos.

Em relação ao perfil socioeconômico, observou-se que a maioria dos idosos é constituída de aposentados (71,9\%), mas 28,1\% declararam ser ativos, desenvolvendo atividades para geração de renda, trabalhando como empregada doméstica/faxineira, lavrador(a), representante comercial, vendedora de roupas e doces e costureira. As ocupações mais citadas foram dona de casa $(34,4 \%)$ e lavrador(a) (18,8\%).

Outro aspecto observado foi a baixa renda familiar desse grupo, predominando a faixa de um a três salários mínimos, constituindo classe econômica abaixo da classe B2, segundo o Critério de Classificação Econômica Brasil,${ }^{15}$ que estima o poder de compra das pessoas e famílias urbanas. A maioria dos idosos $(84,4 \%)$ vive em companhia de esposo(a) ou familiares.

Quando foram perguntados sobre possuir algum problema de saúde, observou-se que 
a hipertensão arterial foi a doença de maior ocorrência (71,9\% dos indivíduos), seguida do diabetes mellitus $(21,9 \%)$ e doenças crônicas da articulação (artrite e artrose) (21,9\%). Gastrite ou presença de úlcera no estômago foram citadas por quatro indivíduos (12,5\%), alterações ósseas (osteoporose e osteopenia), por três idosos $(9,4 \%)$ e alterações graves na visão (glaucoma, catarata e descolamento de retina), também por três indivíduos (9,4\%). Apesar da presença de doença cardiovascular ter sido citada somente por dois indivíduos (6,3\%), é importante destacar que as dislipidemias, um dos principais fatores de risco associados a essa doença, foram citadas por 15 idosos (46,9\%).

\section{0 atendimento nutricional}

Os motivos da procura pelo atendimento nutricional relatados pelos idosos estão representados na figura 1, sendo "indicação médica" o mais citado (17 idosos, 53,1\% da amostra).

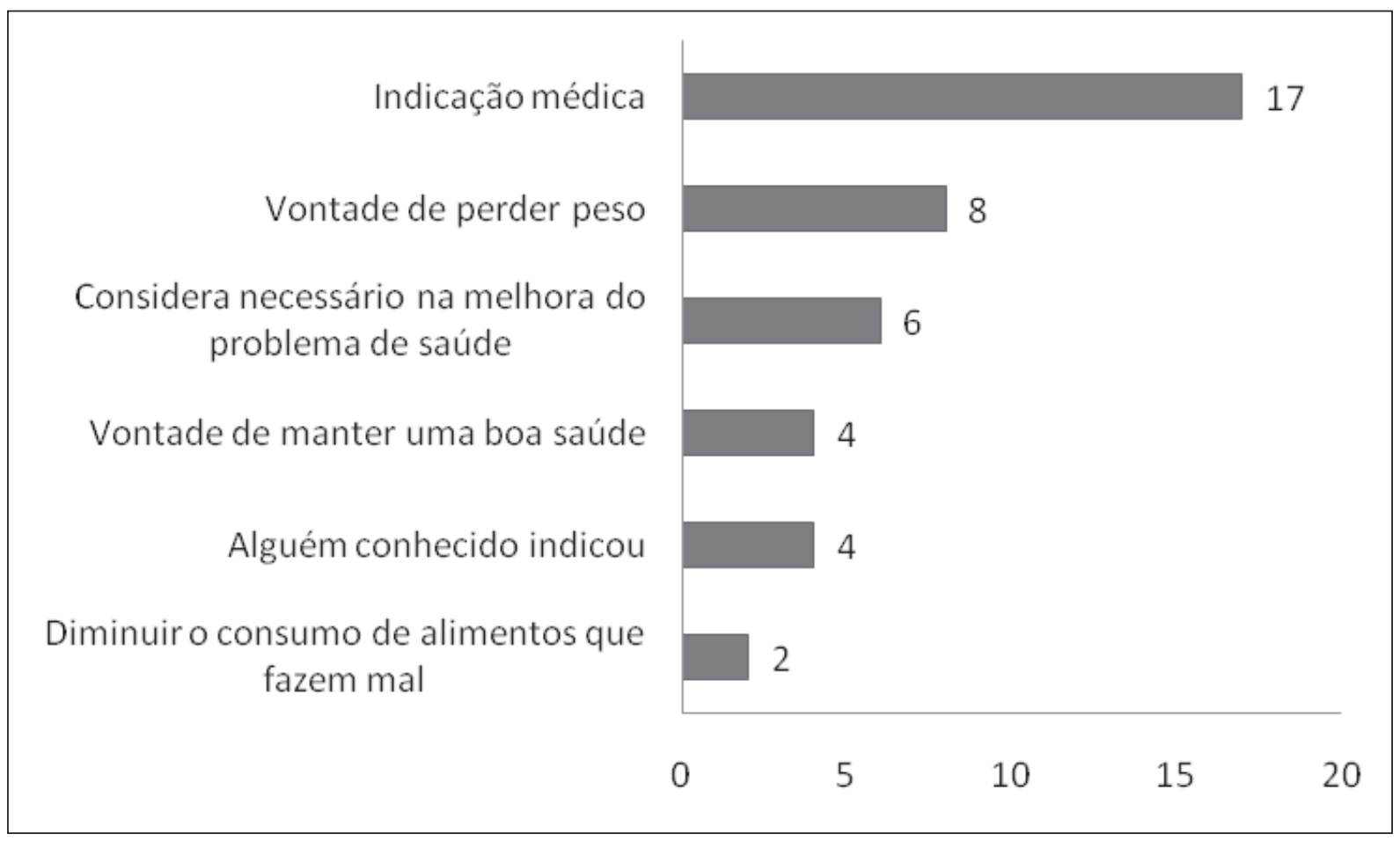

Figura 1 - Motivos da procura pelo atendimento nutricional no PMTI. Viçosa, MG, 2009

Em relação à satisfação dos idosos com o processo do atendimento, este foi classificado como "excelente", "ótimo", "bom” e "razoável", sendo que nenhum indivíduo escolheu as opções "ruim" e "péssimo". Essa classificação está representada na figura 2 . 


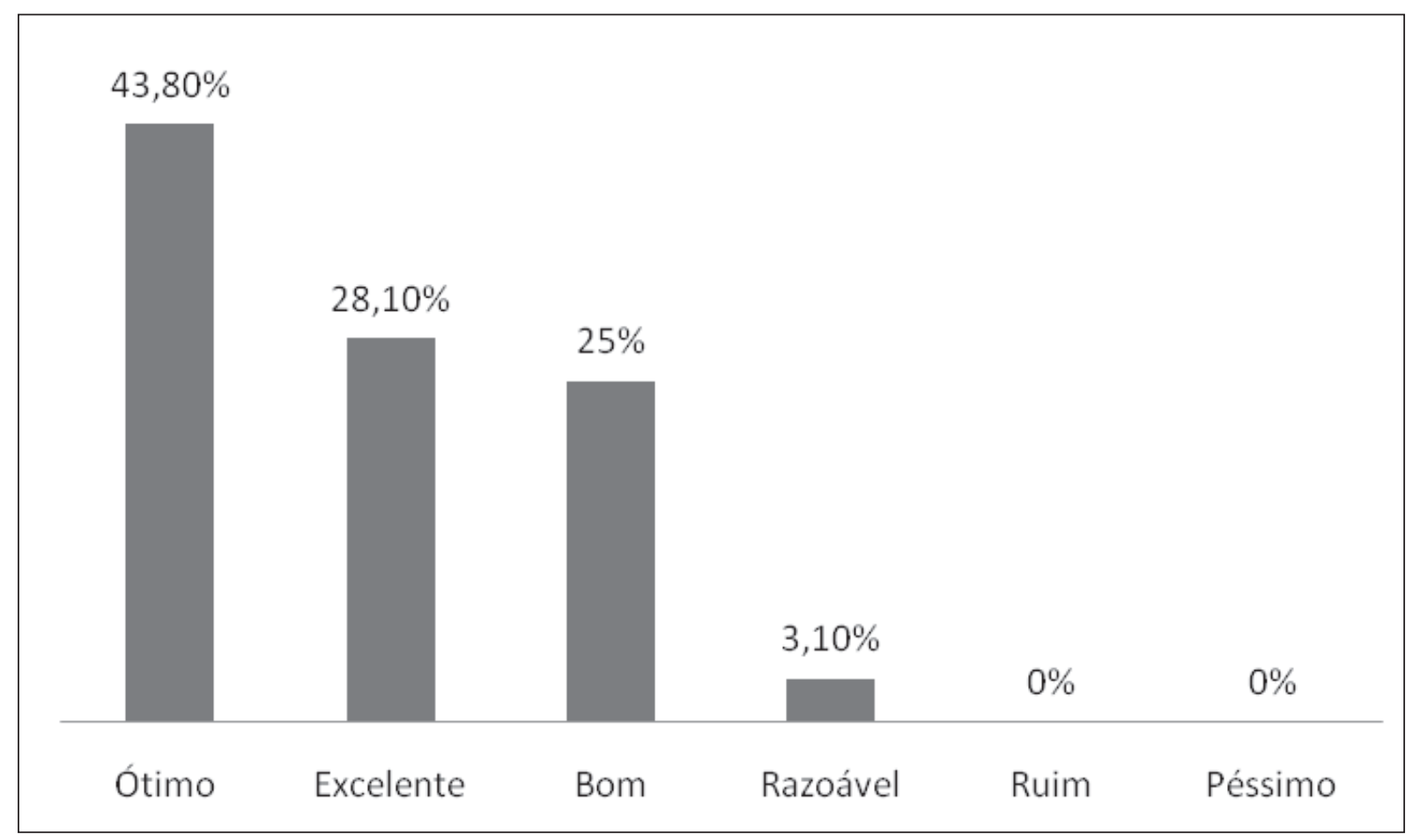

Figura 2 - Classificação do atendimento, por idosos atendidos no PMTI. Viçosa, MG, 2009

Considerou-se "satisfeito" oindivíduoquedeu uma classificação positiva sobre o atendimento, correspondendo ao somatório das respostas "bom", "ótimo" e "excelente". Portanto, 96,9\% da amostra estão satisfeitos com o atendimento e apenas um $(3,1 \%)$ indivíduo, que o considerou "razoável", está parcialmente satisfeito com o atendimento. Ao definir tal classificação, este demonstrou a percepção da pouca experiência de quem o atendia, classificando o(a) atendente como "pouco experiente" e "muito jovem". Da mesma maneira, todos os comentários que expressaram a satisfação com o atendimento foram transcritos abaixo:

Considero ótimo porque ela atende com carinho.

Achei as recomendações dela importantes para a minha saúde.
Toda vida en tive vontade de ir à nutricionista. Em Belo Horizonte eu ia era na endocrinologista. Gostei muito de vir na nutricionista porque, antes, no médico, ele só falava que eu não podia comer muitas coisas, só que ele não falava as quantidades que deveriam ser e 0 que en poderia substituir.

Ela é atenciosa comigo.

A compreensão das recomendações feitas pelos estagiários e nutricionista também foi avaliada e está representada na tabela 1. Diante desse resultado, observou-se que vários idosos não conseguiram compreender integralmente as recomendações.

Em relação ao tempo das consultas, a maioria, 29 indivíduos (90,6\%), achou suficiente e outros três $(9,4 \%)$ acharam necessário mais tempo para esclarecer as dúvidas. 
Tabela 1 - Compreensão dos idosos quanto às recomendações no atendimento nutricional do PMTI. Viçosa, MG, 2009

\begin{tabular}{lcc}
\hline $\begin{array}{l}\text { Compreensão das } \\
\text { recomendações }\end{array}$ & $\mathrm{n}$ & $\%$ \\
\hline Não entendeu nada & 0 & 0 \\
Parcialmente & 9 & 28,1 \\
Sim, tudo & 23 & 71,9 \\
\hline
\end{tabular}

Apenas três idosos disseram que não foram feitas mudanças em sua alimentação, o que se justifica pelo fato de serem pacientes de primeira consulta, cujas modificações são propostas nas consultas posteriores. Dos 29 idosos que disseram ter havido propostas de mudanças em sua alimentação, a maioria considerou não haver dificuldade para realizá-las. As respostas estão representadas na tabela 2 .

Tabela 2 - Existência de dificuldades para adoção das mudanças propostas no atendimento no PMTI. Viçosa, MG, 2009

\begin{tabular}{lcc}
\hline Respostas & $\mathrm{n}$ & $\%$ \\
\hline Sim & 7 & 24,1 \\
Parcialmente & 4 & 13,8 \\
Sim, apenas pelo custo & 2 & 6,9 \\
Não & 16 & 55,2 \\
\hline
\end{tabular}

Alguns idosos relataram quais seriam as dificuldades para adoção de mudanças na alimentação:

Dificuldade de eliminar alguns alimentos que eu já tenho costume. A nutricionista deve cobrar mais para a gente conseguir. Porque nessa idade, estamos cheios de vícios, aí fica mais difícil. Porque se a pessoa não tem interesse e a nutricionista não exige um pouco mais, ela não tem resultado. E o paciente ainda tira a oportunidade de outra pessoa de estar sendo atendida.

A gente não aguenta ficar com aquele pinguinho de comida. A gente já acostumou, né?

Porque exige da gente querer melhorar, exige força de vontade.
Porque manter uma dieta com tudo que você precisa fica mais caro. E fica mais difícil manter aquilo ali dia-a-dia.

Porque com as mudanças sugeridas, vai ter alteração no sabor da comida.

Porque a gente acostumou na roça a comer muita gordura e doces.

Porque en gosto muito de doces. Ela tirou alguns alimentos que eu costumava comer.

A autopercepção do idoso quanto às mudanças ocorridas na saúde e comportamento alimentar após o acompanhamento nutricional está representada na tabela 3. Verificou-se também que a expectativa com a saúde foi positiva, pois $100 \%$ da amostra responderam que a saúde "com certeza" irá melhorar. 
Tabela 3 - Autopercepção de idosos quanto às mudanças na saúde e comportamento alimentar no PMTI. Viçosa, MG, 2009

\begin{tabular}{ll}
\hline Resultados do Acompanhamento & $\mathrm{n}$ \\
\hline Passou a ter mais disciplina & 9 \\
Controle de peso & 7 \\
Melhora da disposição física & 3 \\
Controle da pressão arterial & 2 \\
Passou a consumir alimentos mais saudáveis & 2 \\
Percebeu que as mudanças são necessárias para ter boa saúde & 2 \\
Aumentou a autoestima & 1 \\
Porque ganhou peso & 1 \\
Controle do Diabetes & 1 \\
Controle do colesterol e triglicérides & 1 \\
\hline
\end{tabular}

\section{DISCUSSÃO}

Em relação ao predomínio do sexo feminino sobre o masculino, a maior participação de mulheres em programas voltados para a terceira idade e a maior preocupação destas com a promoção da saúde ocorrem muitas vezes devido à frequência de utilização dos serviços de saúde em momentos anteriores pelas mulheres, além da elevada expectativa de vida para o sexo feminino. ${ }^{4}$

Constatou-se que a maioria dos idosos que são atendidos pelo Serviço de Nutrição vive em companhia de esposo(a) ou familiares, o que pode ser um fator de motivação para eles, na busca pelo atendimento nutricional e consequente cuidado com a própria alimentação. A hipótese de que o convívio familiar influencia no comportamento alimentar também foi levantada em estudo realizado no Japão, ${ }^{16}$ que examinou fatores associados ao apetite em idosos moradores em diferentes comunidades, constatando que a variável "comunicação com a família" foi a única significativamente associada ao bom apetite. Esses pesquisadores destacaram a importância do bom apetite, constatando que os indivíduos que o possuíam eram mais ativos psicologicamente, tinham menos sintomas depressivos e estresse subjetivo, muito mais comunicação com a família e suporte social, quando comparados àqueles que não tinham apetite.

Em estudos epidemiológicos, a morbidade autorreferida é um indicador que tem revelado o perfil de risco da população, sendo a hipertensão arterial e o diabetes doenças que assumiram importância crescente em saúde pública em todos os países. ${ }^{17}$ Estima-se que a hipertensão arterial acomete mais de $50 \%$ da população em idades mais avançadas no Brasil ${ }^{18}$, e agregados a esta condição clínica estão outros fatores de risco e comorbidades responsáveis pela elevada taxa de mortes por doenças cárdio e cerebrovasculares. ${ }^{19}$

Diante da elevada ocorrência dessas doenças nos sujeitos do presente estudo, deve-se enfatizar a importância de intervenções não-farmacológicas, de baixo custo e eficiência no auxílio ao tratamento dessas e outras doenças crônicas. A intervenção nutricional tem merecido destaque, sendo avaliada em pesquisas que demonstraram redução de risco cardiovascular em pacientes 
hipertensos e com excesso de peso; $;^{20}$ e melhoras na adequação das medidas antropométricas e bioquímicas de idosos portadores de diabetes. ${ }^{21}$

Em relação à procura pelo atendimento nutricional, percebe-se através da alta ocorrência de indicação médica, como representado na figura 1, que no PMTI as intervenções na saúde do idoso são de caráter multiprofissional. Essa característica demonstra que a proposta do programa reflete as atribuições da gerontologia, uma ciência que estabelece a necessidade de atuação de múltiplas áreas de competência. ${ }^{22}$ Além do motivo "indicação médica", outras razões foram associadas à procura pelo atendimento nutricional, sendo elas reflexo de características da maioria dos sujeitos deste estudo: indivíduos com autonomia e que reconhecem no serviço nutricional sua importância na melhoria do problema de saúde ou como instrumento para alcance de metas pessoais (como perda de peso e diminuição do consumo de alimentos que fazem mal à saúde).

A percepção dos usuários sobre a qualidade do serviço pode ser analisada por meio da medida de satisfação. Essa é uma avaliação pessoal dos cuidados e dos serviços de saúde oferecidos. ${ }^{23}$ Estudos sobre a satisfação mostram que, geralmente, os usuários estão mais satisfeitos com os aspectos relacionais (atendimento, cuidado, comunicação interpessoal e outros) do que os organizacionais (estrutura física, acesso a consultas e medicamentos). ${ }^{24,25}$ Tais constatações levam a crer que o sucesso final do tratamento pode ter forte relação com o estilo de comunicação entre profissional e paciente, sendo este aspecto predominante sobre a qualidade técnica no transcorrer da consulta, sobretudo após ter sido feita a escolha ou indicação do profissional. ${ }^{26}$

Foi possível observar, por meio do relato do(a) idoso(a) que percebeu a pouca experiência de quem o atendia, que o usuário de um serviço para a terceira idade pode ser um observador crítico do comportamento de quem o atende. Essa insatisfação em relação à pouca experiência do atendente exige o cuidado de elaborar treinamentos prévios, durante período prolongado, com consentimento do paciente, para que o estagiário adquira segurança e se habitue com a rotina de atendimento.

Apesar dessa constatação, cabe ressaltar a importância de desenvolver precocemente a inserção do aluno em atividades cotidianas nos serviços para identificação das necessidades de saúde, desenvolvendo sua responsabilidade social. ${ }^{27}$

O exemplo do atendimento nutricional realizado no PMTI é positivo, pois permite aos idosos receber atenção individualizada, possibilitando a formação de vínculo entre paciente e atendente, como expresso no primeiro e último discurso dos idosos. Além disso, o atendimento individualizado e focado na alimentação permite maior detalhamento das ações propostas pelo(a) nutricionista/estagiário(a), que resultará em maior esclarecimento para o idoso, como demonstrado no segundo e terceiro discursos. Por outro lado, na perspectiva do estagiário, a oportunidade permite o treinamento dos conhecimentos teóricos e práticos, bem como lidar com o paciente e suas expectativas, que devem ser foco da atuação no serviço prestado.

Observou-se nas falas que expressaram a satisfação que, sendo essa uma característica bastante subjetiva, neste grupo se relacionou muito mais a aspectos de cuidado, atenção com o paciente e capacidade de esclarecer as dúvidas do que com aspectos organizacionais (estrutura física, acesso, custo) e as técnicas utilizadas na consulta, confirmando o que foi constatado por outros autores, que estudaram os fatores que influem na satisfação do usuário de um serviço de saúde. ${ }^{24}$

Diante do resultado da pouca compreensão das recomendações nutricionais por alguns idosos $(28,1 \%)$, é preciso destacar a existência, na literatura internacional, de determinações de competências em geriatria e gerontologia, que devem ser desenvolvidas na graduação. Elas 
sistematizam atitudes, preconizando treinar o aluno em diversas habilidades que incluem a capacidade de comunicação com o idoso, mesmo em presença de déficits sensoriais no paciente. ${ }^{28,29}$

A necessidade de mudar hábitos foi a maior dificuldade percebida por esses idosos, seguida do custo de se manter uma alimentação completa em nutrientes adequados. Diante desses relatos, deve-se destacar o papel do nutricionista como o profissional habilitado a realizar um trabalho contínuo com o paciente, de estímulo a mudanças de hábitos e de adequações dos planos alimentares às condições econômicas do grupo, sem prejudicar o valor nutricional das refeições prescritas. Algumas estratégias para diminuir o custo e não prejudicar o valor nutricional das refeições são: dar preferência a alimentos como frutas e verduras, principalmente aqueles que estão no período de safra e portanto têm menor custo; estimular a formação de hortas nas casas onde isso é possível; investigar os preços dos produtos adquiridos e sugerir opções de compras; ensinar técnicas de aproveitamento integral dos alimentos, higiene alimentar e porcionamento adequado dos alimentos.

Todas as mudanças positivas reconhecidas pelos idosos do PMTI como resultado do acompanhamento nutricional reforçam o perfil desse grupo, constituído de pessoas que buscam envelhecer de maneira ativa, prevenir e controlar doenças que surgem nessa idade, ter disposição física e melhorar a autoestima.

Em relação à expectativa positiva sobre a saúde, demonstrada por todos os idosos após o atendimento, pode-se inferir que a intervenção nutricional influenciou positivamente na maneira de pensar dos idosos sobre alimentação e saúde.

No entanto, duas limitações observadas neste estudo devem ser consideradas. A primeira refere-se ao pequeno tamanho amostral, que não reflete a totalidade de idosos atendidos ao final do período de um ano, mas corresponde a todos os indivíduos que foram atendidos em um semestre completo de atividades do programa. A segunda diz respeito à impossibilidade de generalização dos resultados, que é característica de pesquisas qualitativas. Não obstante, esta abordagem qualitativa se faz essencial à medida que fornece elementos como ponto de partida para orientar as práticas adotadas no serviço analisado, bem como para direcionar futuros trabalhos de avaliação do mesmo e de outros serviços.

\section{CONCLUSÃO}

O grupo estudado é formado por indivíduos de classe econômica baixa, em sua maioria portadores de doenças crônicas nãotransmissíveis, mas que usufruem de maneira ativa o serviço oferecido por um programa que, apesar de possuir características singulares, é um espaço de convivência social e de atuação multiprofissional/multidisciplinar para a promoção da saúde.

Em relação à percepção sobre qualidade e resultados obtidos com o acompanhamento nutricional, os usuários declararam compreendêlo como instrumento de auxílio na busca de melhoria na qualidade de vida e associaram a satisfação com o serviço a aspectos de cuidado e atenção.

Estimular a formação de programas governamentais que reúnam indivíduos com este perfil é uma forma de fortalecer vínculos entre eles, repassando aos idosos que ainda não desenvolveram essa iniciativa os benefícios de se ter uma alimentação saudável. Além disso, o estímulo a pesquisas com grupos e programas de nutrição na terceira idade também se torna cada vez mais necessário, para que se busquem qualidade nutricional e melhores estratégias de atendimento aos idosos.

\section{AGRADECIMENTOS}

Aos idosos voluntários que, de maneira acolhedora, colaboraram com este trabalho. 


\section{REFERÊNCIAS}

1. Najas MS, Andreazza R, Souza ALM, Sachs A, Guedes ACB, Sampaio LR, et al. Padrão alimentar de idosos de diferentes estratos socioeconômicos residentes em localidade urbana da região sudeste, Brasil. Rev. Saúde Pública. 1994; 28 (3): 187-91.

2. Silva MLT. Geriatria. In: Waitzberg DL. Nutrição oral, enteral e parenteral na prática clínica. São Paulo: Atheneu; 2000. p.997-1008.

3. Campos MTFS, Coelho AIM. Alimentação saudável na terceira idade: estratégias úteis. 2.ed. Viçosa: UFV; 2005.

4. Cervato AM, Derntl AM, Latorre, MRDO, Marucci MFN. Educação nutricional para adultos e idosos: uma experiência positiva em Universidade Aberta para a Terceira Idade. Rev Nutr. 2005; 18(1): 41-52.

5. World Health Organization. WHO expert Committee on Physical Status: the use and interpretation of antropometry physical status: the use and interpretation of anthropometry. Report of a WHO Expert Commitee. Switzerland; 1995.

6. Kottow M. Fundamentos de Bioética. In: Introdución a La Bioética. Santiago: Editorial Universitária; 1995. p.72-6.

7. World Health Organization. Envelhecimento ativo: uma política de saúde. Brasília: Organização PanAmericana da saúde; 2005.

8. Jardim VCFS, Medeiros BF, Brito AM. Um olhar sobre o processo do envelhecimento: a percepção de idosos sobre a velhice. Revista brasileira de geriatria e gerontologia.2006; 9(2): 25-34.

9. Silva CA, Fossatti AF, Portella MR. Percepção do homem idoso em relação às transformações decorrentes do processo do envelhecimento humano. Estudos Interdisciplinares sobre o Envelhecimento 2008; 12: 111-26.

10. Vuori H. Research needs in quality assurance. Qual Assur Health Care 1989; 1 (2-3): 147-59.

11. Akerman M, Nadanovsky P. Avaliação dos serviços de saúde: avaliar o quê? Cad Saúde Pública.1992; 8 (4): 361-5.

12. Bosi MLM, Affonso KC. Cidadania, participação popular e saúde: com a palavra, os usuários da Rede Pública de Serviços. Cad Saúde Pública. 1998; 14 (2): 355-65.

13. Minayo MCS, Souza ER, Assis SG, Cruz Neto O, Deslandes SF, Silva CMFP. Avaliação dos Centros de Orientação e Apoio Sorológico/CTA/Coas da região nordeste do Brasil. Cad Saúde Pública. 1999; 15 (2): 355-67.

14. Nuto SS, Nations MK. Avaliação qualitativa dos serviços como processo de construção de cidadania. Ação Coletiva. 1999; 2:25-9.

15. Associação Brasileira de Empresas de Pesquisa (ABEP). Critério de Classificação Econômica Brasil. São Paulo: ABEP; 2003. Disponível em: URL: http://www.abep.org/codigosguias/CCEB2008Base2006e2007.pdf.

16. Okamoto K, Harasawa Y, Shiraishi T, Sakuma $\mathrm{K}$, Momose Y. Much communication with family and appetite among elderly persons in Japan. Arch Gerontol Geriatr. 2007; 45 (3): 319-26.

17. Brasil. Ministério da Saúde. Vigilância de fatores de risco e proteção para doenças crônicas por inquérito telefônico. Brasília: Ministério da saúde; 2008.

18. Brasil. Ministério da Saúde. Plano de reorganização da atenção à hipertensão arterial e ao diabetes mellitus: hipertensão arterial e diabetes mellitus. Brasília: Ministério da Saúde; 2001.

19. Zarnke KB. Recent developments in the assessment and management of hypertension: SHEP, ALLHAT and LIFE. Geriatrics \& Aging 2003; 6 (2):14-20.

20. Alvarez TS, Zanella MT. Impacto de dois programas de educação nutricional sobre o risco cardiovascular em pacientes hipertensos e com excesso de peso. Rev Nutr. 2009; 22 (1): 71-9.

21. Geraldo JM, Alfenas RCG, Alves RDM, Salles VF, Queiroz VMV, Bitencourt MCB. Intervenção nutricional sobre medidas antropométricas e glicemia de jejum de pacientes diabéticos. Rev Nutr. 2008; 21(3): 329-40.

22. Papaleo Netto M, Yuaso DR. Interdisciplinaridade em Gerontologia: aspectos conceituais e objetivos. In: Papaleo Netto M. Tratado de Gerontologia. São Paulo: Atheneu; 2006.

23. Ware JE. Defining and measuring patient satisfaction with medical care. Eval Program Plann 1983; 6 (3-4): 247-63.

24. Trad LAB, Bastos ACS, Santana EM, Nunes MO. Estudo etnográfico da satisfação do usuário do Programa de Saúde da família (PSF) na Bahia. Ciências \& Saúde Coletiva. 2002; 7(3): 581-9.

25. Gross R, Tabenkin H, Porath A, Heymann A, Greenstein M, Porter B, et al. The relationship between primary care physicians' adherence to 
guidelines for the treatment of diabetes and patient satisfaction: findings from a pilot study. Fam Pract. 2003; 20 (5):563-9.

26. Buller KM, Buller BD. Physicians communication style and patient satisfaction. J Hlth Soc Behav. 1987; 28 (4): 375-88.

27. Motta LB, Aguiar AC. Novas competências profissionais em saúde e o envelhecimento populacional brasileiro: integralidade, interdisciplinaridade e intersetorialidade. Ciências \& Saúde Coletiva. 2007; 12(2): 363-72.

28. Education in geriatric Medicine. AGS Education Committee and Public Policy Advisory Group (PPAG). J Am Geriatr Soc. 2001; 49 (2): 223-4.

29. Care management position statement. J Am Geriatr Soc. 2000; 48:1338-9.

Recebido: 26/5/2011

Revisado: 29/11/2011

Aprovado: 13/12/2011 